\title{
Autonomous Decentralized Community Construction Technology to Assure Quality of Services
}

\author{
Takanori Ono, Naohiro Kaji, Yuji Horikoshi, \\ Hisayuki Kuriyama, Khaled Ragab and Kinji Mori \\ Tokyo Institute of Technology \\ 2-12-1 Ookayama, Meguro, Tokyo 152-8552, Japan \\ Tel: +81-3-5734-2664, Fax: +81-3-5734-2510 \\ Email: \{nouri@mori., nkaji@mori., horikoshi@mori., kuriyama@mori., \\ ragab@mori., mori@\}cs.titech.ac.jp
}

\begin{abstract}
Recent advances in the wireless communication and mobile telecommunications have made mobile commerce possible. In the retail business under the evolving market, the users solicit continuously to utilize the appropriate services based on their preferences and situations. In addition, the service providers insist on seizing the current requirements of the majority of the users in the local service area in order to customize the service for the current local majority of the service area. However, these local and well-customized services cannot be realized through global information services on the Internet. In addition, under a constantly changing market the situation-aware service is one challenge facing further developments of the m-commerce. Service-Oriented Community System (SOCS) is a proposition made to ride out this challenge. It is proposed to satisfy both users' and service providers' requirements. The community members, users and service providers, mutually cooperate to utilizelprovide three-dimensional service with awareness of the user's situation (location, time and demand).

We identify that the reliability of the communication among the community members is an important issue to assure the quality of the community service. Thus, the contribution of this paper is the proposition of the autonomous decentralized construction technology to assure the quality of services to the community members. The effectiveness of the proposed technology is shown through the simulation.
\end{abstract}

Key words: Service-oriented Community, Situation-aware Services and Autonomous Decentralized System.

\section{Introduction}

The widespread deployment and use of wireless data communications is generally recognized as being the next major advance in the information technology industry. Wireless data network represents a key enabling technology underlying the vision of Location Based Service (LBS). It is the ability to find the geographical location of the mobile device and provide services based on this location information $[1,2,3]$. The emergence of mobile devices made the situation-aware services particularly interest. We identify situation-aware services to be not only location-aware but also time-aware services and demand-aware services. In other words, the situation-aware services should address the scope of awareness regarding to the users' locations, time and preferences. Under constantly changing market the situation-aware service is one of the challenges facing further developments of the m-commerce. We have overcome this challenge by proposing service-oriented community system [7]. Two actors coexisted in this system: Service Provider (SP) offers the service and mobile-users consume the service and we called them community members. They mutually cooperate to utilize/provide three-dimensional service with awareness of the user's situation (location, time and demand). We argue that the long run of this system satisfies both, the user's requirements as well as the service provider's requirements to the situation awareness service. Along the run of this system the reliable communication among the community members is highly required to achieve the quality of service they required. We identify that the growth of the community system may affect the reliability of the communication among the community members. Thus, the main contribution of this paper is the proposition of the autonomous decentralized construction technology to assure the quality of the community service.

This paper is organized as follow. Section 2 clarifies the service-oriented community system concept and exhibits both system architecture and community communication processes. Section 3 exposes the proposed technology. Section 4 presents simulation results showing reliability improvement. The last section draws conclusion. 


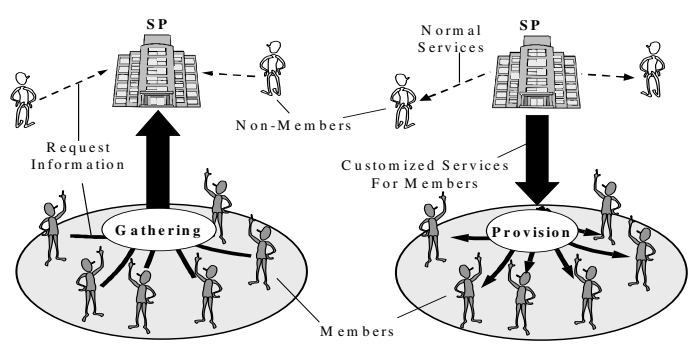

Figure 1: Community Services

\section{Service-oriented Community: Concept and System Architecture}

\subsection{Concept}

The main concern of the conventional m-commerce information systems have been in the past to provide services based on the location information [3]. In such systems, SP provides the service regardless of the mobile-users' demands and situations. We identify the situation-aware service to be not only location-aware, but also time-aware and demand-aware service. The current systems have the ability to find the geographical location of the mobile device [5] and provide services based on this location information. There is no discernment between differences in time; mobile-users in any situations receive the same contents. Moreover, in these systems users are passive actors they only consume the service. As a result the SP cannot provide a well-customized service.

As the mobile-users' demands and situations are dynamically changing, somewhere at specified time there are significant numbers of users' sharing the same demands and situation. We introduced a new term called local-majority in which the majority of the mobile-users in specified area (somewhere) at specified time (sometime), share the same demands (someone). We have proposed Service-oriented Community concept [7] to provide three-dimensional service with awareness of the user's situation (location, time and demand). This concept brings a new consequence to the mobile-users and makes them cooperative and active actors. The mobile users and the service providers in that area are called the community members. Those community members bid their preference information to the appropriate service providers based on their situations and meanwhile service providers apply the marketing information in the local area and customize the service to the local-majority (mobile-users) in that area as shown in figure 1 . In addition, the mobile-users in that area cooperate to drive down prices based on their aggregated purchasing power.

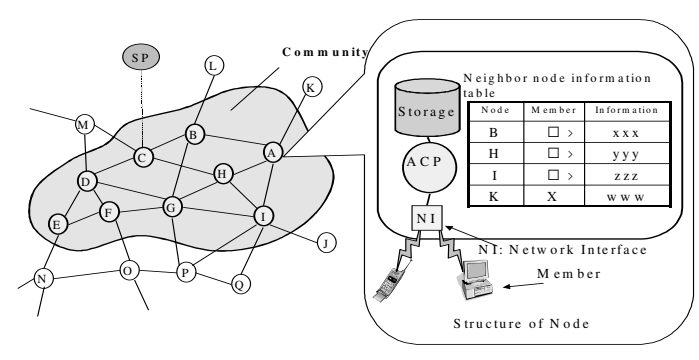

Figure 2: Community System Architecture

\subsection{System Architecture}

The autonomous service-oriented community system is structured based on the Autonomous Decentralized System (ADS) concept [6]. It is proposed to foster the concept of the service-oriented community. It is a set of autonomous subsystems: nodes, SP and user's mobile devices. Nodes are base stations of the wireless network. Figure 2 shows the community network. Each node is responsible for a physical area called cell. Each node has Autonomous Control Processor (ACP); it is responsible to judge autonomously based on its local information. The local information such as users' demands, services' information and neighbors node information table are stored in its own storage. Each node receives a message and then broadcasts it to the users in its cell. Each node keeps track of the time distance to its immediate neighbors in a table. The propagated message format is shown in figure 3 . We have introduced the Time Distance as a logical distance that defines the user's physical moving time [7,8]. It has been proposed to determine the service area. The time distance is used instead of the physical distance because of the user's access to the SP is differently based on the physical conditions (such as a river, or some physical obstacles, and traffic jam). Time distance is an efficient measure of the distance from users to the SPs with considering the users' situation such as the traffic jam that he/she faced.

\section{\begin{tabular}{|l|l|l|}
\hline CC & Sender & Service Property \\
\hline
\end{tabular} Figure 3: Message Format}

Service provider typically communicates with its nearest physical node (base station) to provide its services. Each service has a lifetime such as, the expiration time of the product. The SP sets the appropriate service lifetime according to the service characteristics. The proposed service-oriented community system is responsible for distributing the information of the service to the users and then gathers the marketing information in the appropriate service area based on the service lifetime. This area, we called it a community service area. Moreover, the users have a wireless connection to the nearest nodes and provide their preferences and demands to the system for customizing the service.

In our previous research [7,9], the role of the serviceoriented community system is to timely send the right 
information to the right user. Obviously, gathering process of the users preferences and service information provision process must be reliable. Thus, we identified that reliable communication among the community members is an important issue that should be investigated. In this paper, we address this issue by proposing the construction technologies in order to provide a reliable communication service among the community members.

\subsection{Communication Processes in Community}

Mobile-users in the current m-commerce information systems are passive actors they only consume the service. As a result the SP cannot provide a well-customized service. Typically, most of the services providers need to increase their sales constantly regardless of the evolving market. In the retail business, SP provides the service for users in the local area around it not a global one. In addition, it has to customize the service to the users in the local service area. Therefore, SP has to grasp the users' marketing information not only timely but also reliably.

We have proposed two technologies: Autonomous Information Provision Technology [7] and Autonomous Synchronization Technology [9]. The first technology is made to achieve flexibility of the information provision based on services. It has the following scenario: SP sends a request message for announcing its service to the community service area. This area based on the service lifetime. In the other hand, the autonomous synchronization technology is proposed to collect the user's marketing information timely. The collecting process of the users' marketing information has the two phases. First, SP receives the user's information. Second, it counts the users requirements. Figure 4 shows the information provision process and the gathering information process in the community system.

\subsubsection{Problem of Reliability in Communication}

The communication in the community has the two phases as shown in figure 4 and illustrated as follows:

- Communication between community and SP: Figure 4-a shows that there is one community node A that is responsible to communicate with the SP. This node is in charge of forwarding the gathered information from the community area to SP. In addition, it is responsible to forwards SP' service information to the community area as shown in figure 4-b. Obviously, this node acts as a single point of failure and makes the communication is less reliable.

- Communication within community area: The autonomous decentralized community communication technology has been proposed for productive cooperation among community members [8]. When a communication path between community nodes is down, the other community nodes cooperate to avoid this failure by making an alternative path [9].

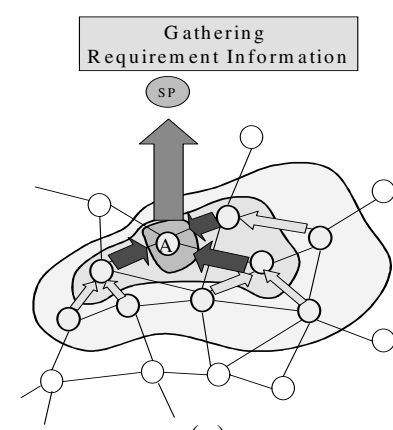

(a)

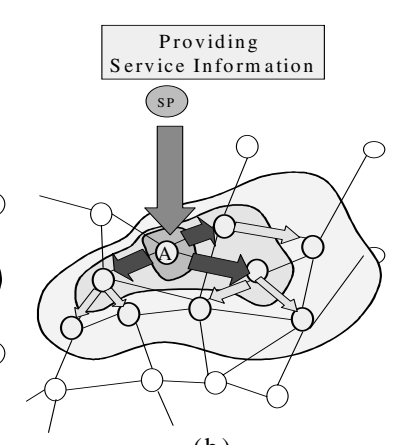

(b)
Figure 4: Information provision and gathering processes

It is obvious that if there are many alternative routes, the reliability of the community communication is increased. Thus, more members and more links provide more alternative paths and so the reliability of the community communication is improved.

Next section presents the proposed autonomous decentralized construction technology for achieving the reliability of the community service.

\section{Autonomous Decentralized Community Construction Technology}

\subsection{Goals and Requirements}

The first goal is to construct the community service-oriented network that can support information provision and gathering processes reliably. In addition, we should avoid hotspots (single point of failure) in the community network by organizing the community network into sub-communities. Finally, the community network has to provide redundancy. Node failures must not lead to the community network disconnected or severely hampering the reliability of the provision/gathering processes.

\subsection{Organizing Community Network}

In this section, we will organize the community network into sub-communities. Each sub-community has a leader that is responsible to communicate with SP for both information provision and gathering processes. Thus, we avoid the single point of failure that could be happening as shown in the previous section and figure 4. All members (base stations) in the sub-community can communicate with the SP through leaders. We thought this solution would improve the reliability of the community communication because SP and other community members can receive redundancy of information in parallel. The members in one sub-community communicate together based on an extended message format as shown in figure 5. It contains new field that represents the Sub-community identifier. Each member registers the SubCommunityID (SCID) in its ACP that is corresponding to its sub-community. Thus, when a node receives a message that has SCID, it checks if 
the received message SCID is same with its SubCommunityID or not. There are two cases as shown in figure 6 and illustrated as follows: If it is same then it forwards this message to its neighbors except the sender of that message. Otherwise, it means that the message from other sub-community then it discards that message.

\section{\begin{tabular}{|l|l|l|l|} 
CC & SCID & Sender & Service Property \\
\hline
\end{tabular}}

Figure 5: Extended Message Format

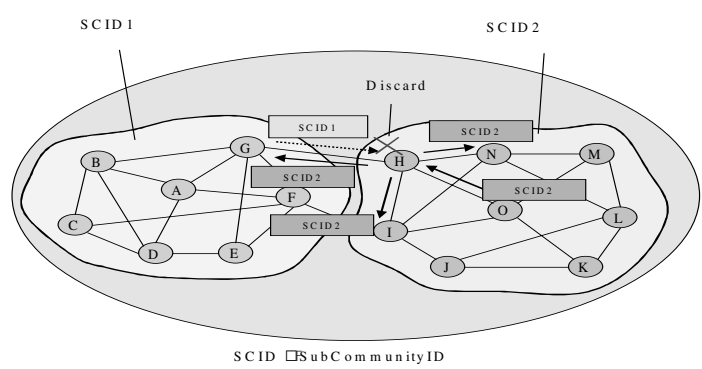

Figure 6: Sub-communities communication

We thought that organizing the community network into sub-communities enforces us to present a corresponding reliability model of the community communication in next subsection.

\subsection{Reliability Model}

The reliability model of the communication in the community that is organized into sub-communities is shown in figure 7 and will be illustrated as follow.

The reliability model depends on three variables $R_{1}, R_{2}$ and $\mathrm{R}_{3}$.

- The reliability of the communication between SP and the community is $\mathrm{R}_{1}$. As the number of the sub-communities increases, the number of alternative routes between community and SP increased too. Therefore, the increase of dividing times of the community into sub-communities will improve the reliability of the communication between SP and the community.

- $\mathrm{R}_{2}$ is the reliability of the communication within a sub-community. As the number of members in the sub-community increases, the number of the alternative routes between member nodes and the leader node increase too. Therefore, as the number of members in the sub-community increases, the reliability $\mathrm{R}_{2}$ is improved. It is defined as follows:

$$
R_{2}=1-\left(1-r_{2}\right)^{\alpha\left(\begin{array}{l}
n \\
2
\end{array}\right)}
$$

Where, $\mathrm{n}$ is the number of members in the sub-community, $r_{2}$ is the reliability of the communication between two community members and $\alpha\left(\begin{array}{l}n \\ 2\end{array}\right)$ is the number of alternative routes $(0<\alpha<1)$.

- $\mathrm{R}_{3}$ is the reliability of the communication between sub-communities. It is depend on the number of nodes on the path between the leader of a sub-community and the other one. We assume that more members in a sub-community lead to the situation that there are more members in the path between two leaders of two close sub-communities. Thus, the reliability between two sub-communities comes down. Assuming $\beta_{n}$ is the number of members that are on the path between two leaders. Thus, $\mathrm{R}_{3}$ can be written as follows:

$$
R_{3}=r_{2}^{\beta_{n}}
$$

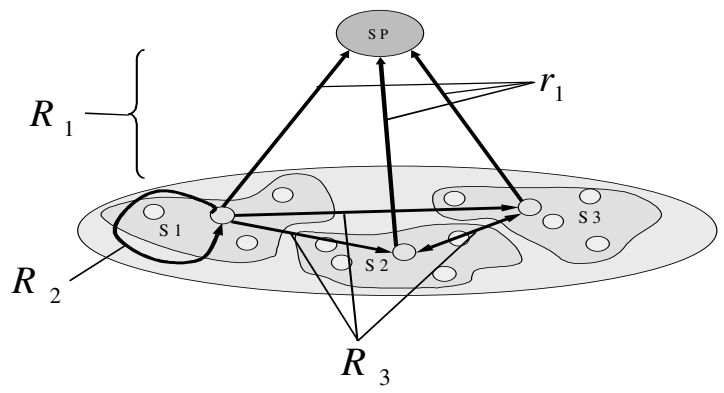

Figure 7: Reliability Model

Thus, the reliability of the community communication can be defined as follows:

$$
R=\left\{1-\prod_{k=0}^{N_{s}-1}\left(1-R_{3}^{k} r_{1}\right)^{N_{s} C_{2}}\right\} \times R_{2}
$$

Where $r_{1}$ is the reliability of the communication between leader and SP. $\mathrm{N}_{\mathrm{s}}$ is the number of sub-communities. Figure 8 shows an example of the relationship between the number of the sub-communities and the reliability $\left(r_{1}=0.8, r_{2}=0.7\right.$, $\alpha=\beta=0.1$ ). This figure plots the variation of the reliability with the number of the sub-communities. It shows that the reliability increases with the increasing of the number of the sub-communities until reach a peak point and then the reliability will be descended. It depicts the following conclusion. The reliability does not increase as long as the number of the sub-communities is increased. Therefore, it is highly required to have the reconstruction technologies that increase and decrease the number of the sub-communities to improve the reliability. Those technologies will be shown in the next subsection 3.4.

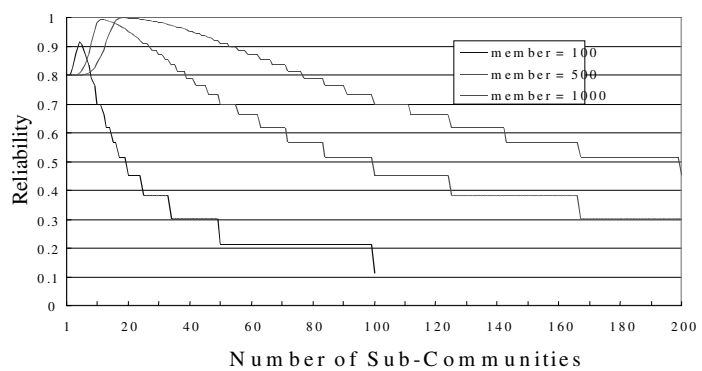

Figure 8: The relationship between the number of sub-communities and the reliability. 


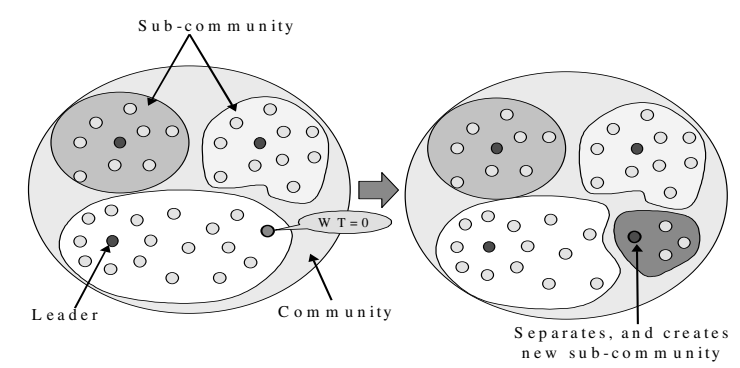

Figure 9: Division Process

\subsection{Proposed Technologies}

The community network is organized into sub-communities to improve the reliability of the community services. This section illustrates four technologies that are proposed to improve the reliability of the community services. The main goal of these technologies is to adapt the reliability of the community service with the changing of the number of members of the community.

\subsubsection{Autonomous Division Technology}

Each community node (member) periodically exchanges keep-membership message with the leader of the sub-community. If a member does not receive that message for a period of time $W T_{s u b}$, It behaves as follows:

1. Creates new sub-community that has SCID equal to its member ID and becomes a leader of this sub-community.

2. Sends an invitation message to its neighbors in order to join its own sub-community.

3. Then, it sends the keep-membership messages to all neighbors periodically.

Thus, when a leader fails or a network path is down, the other members judge autonomously to create their own sub-communities and continue their management as shown in figure 9. In case of two neighbors create a sub-community at the same time; one of them promotes the leadership to the other one.

\subsubsection{Autonomous Fusion Technology}

The community nodes leave and join dynamically. Thus, the size of two close sub-communities becomes small and the path between the leaders becomes short too. Therefore, the number of the sub-communities increases and leads to degradation of the reliability. Thus, the fusion technology is required to reduce the number of the sub-communities as shown in figure 10. Each leader determines the number of hops (HOPS) to its neighbor sub-community and then compares it with threshold $\mathrm{Sub}_{\max }$. If $H O P S \geq S u b_{\max }$ then the leader continues to be a leader of its sub-community. Otherwise, if $\mathrm{HOPS}<\mathrm{Sub}_{\max }$ then the leader judges that the distance to its neighbor sub-community is short and has to merge with its neighbor sub-community. Therefore, the leader behaves as following steps:

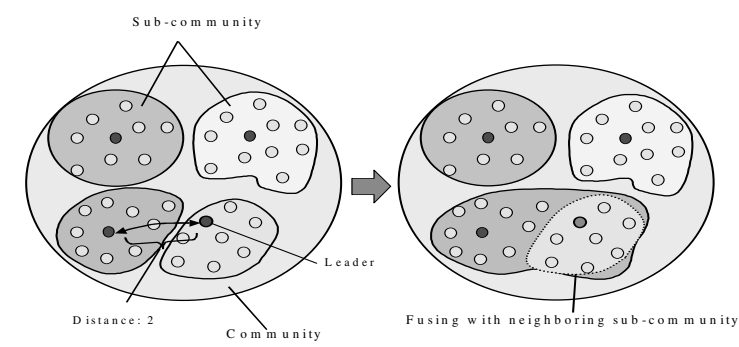

Figure 10: Fusion Process

1. It becomes a normal member and sets SCID to its neighbors sub-community ID.

2. Sends message to the other sub-community members informing them to change their SubCommunityID to the new SCID.

\subsubsection{Autonomous Transition Technology}

Each member can transfer from a sub-community to another one in order to improve the reliability of the communication as shown in figure 11. Each member can determine the maximum distance $\left(\mathrm{HOPS}^{(\mathrm{i})}{ }_{\max }\right)$ and minimum distance (HOPS ${ }^{(i)}{ }_{\text {min }}$ ) to the leader of the closest sub-community with SubCommunityID equals i. Moreover, each member can determine the distance D to its leader. Then, each member executes the following instance of code:

Procedure Transfer(self, SCID)

[// Transfer the calling member (self) to the neighboring // sub-community that has SCID.

$$
\begin{aligned}
& \text { If }\left(\left(\mathrm{D}<\mathrm{HOPS}^{(\mathrm{SCID})} \max _{\text {ax }} \text { and HOPS }{ }_{\text {max }}^{(\mathrm{SCID})}<\mathrm{Sub}_{\max }\right)\right. \\
& \text { Or } \left.\left(\mathrm{D} \geq \operatorname{HOPS}^{(\mathrm{SCID})} \min \right)\right) \\
& \text { Self } \rightarrow \text { SubCommunityID:= SCID; }
\end{aligned}
$$$$
\text { \} }
$$

The expression (Self $\rightarrow$ Var) means that we seek the value of Var from the calling member (self). This procedure allows a member to judge if the neighboring sub-community is more appropriate than the current one or not. If it is appropriate, member sets its SubCommunityID into neighboring SubCommunityID. Thus, this member registers the neighboring SubCommunityID (SCID) in its ACP and then receives any message that contains this SCID.

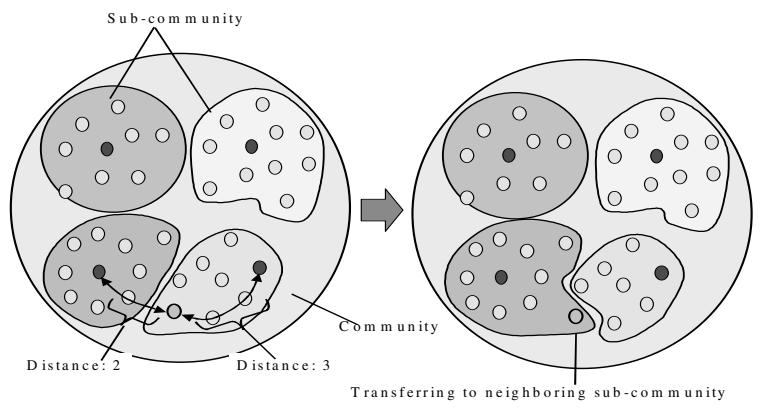

Figure 11: Transition Process 


\subsubsection{Autonomous Adaptation Technology}

The reliability of the community communication can be improved if we adjust the sub-community size. This section illustrates the required technology to adjust the sub-community size. The scenario of this technology is as follows. Each member grasps the current size of the whole community and its sub-community as well. Then, it changes the parameters that have been used in the three technologies have been mentioned before in order to adapt the changing situations. The frequencies of receiving community messages and sub-community messages are used as parameters to grasp the situations on the premise that the frequency is proportionate to the sizes. In this adaptation technology, each member counts the number of received community messages $\left(\mathrm{cnt}_{\mathrm{com}}\right)$ and the number of received messages from its sub-community ( $\left.\mathrm{cnt}_{\text {sub }}\right)$. Then periodically every $\mathrm{T}_{\text {cycle }}$, each member calculates the ratio $\mathrm{r}$.

$$
r=\left\{\begin{array}{cc}
\frac{c n t_{\text {sub }}}{c n t_{\text {com }}} & \left(c n t_{\text {com }} \neq 0\right) \\
1 & \left(c n t_{\text {com }}=0\right)
\end{array}\right.
$$

Assuming that the floor and ceiling $\left(\varepsilon_{L}, \varepsilon_{U}\right)$ are given for the frequency $r$. Each member can judge its sub-community small, large or appropriate as follows. The sub-community size is small when $\left(r<\varepsilon_{L}\right)$. The sub-community is large if $\left(\varepsilon_{U}<r\right)$. Otherwise, the sub-community size is appropriate and no need to change the parameters, if $\left(\varepsilon_{L} \leq r \leq \varepsilon_{U}\right)$ Whereas, in the first and second cases, the parameters $\mathrm{WT}_{\text {sub }}$ and $\mathrm{Sub}_{\max }$ must be changed as follows to increase the sub-community size:

- $W T_{\text {sub }}$. The waiting time for a message from a leader should be long to increase the size of the sub-community.

- $\mathrm{Sub}_{\max }$. Each member can transfer to its neighbor sub-community when it is far from the leader of the neighbor sub-community. Thus, increasing the $\mathrm{Sub}_{\max }$ is required to allow the member to transfer to the neighbor sub-community.

Therefore, we conclude that to enlarge/condense the sub-community size, it is required to increase/decrease both $\mathrm{WT}_{\text {sub }}$ and $\mathrm{Sub}_{\max }$. In addition, the coordination among the community members can form the sub-community by changing the parameters to adapt the changes and achieve the high reliability of the community communication with appropriate thresholds (section 3.3). Next section shows the simulation results of the previously mentioned technologies.

\section{Simulation}

The main goal of this simulation is to verify the behavior of the community system to adapt the changes and to evaluate the reliability of the community communication as well.
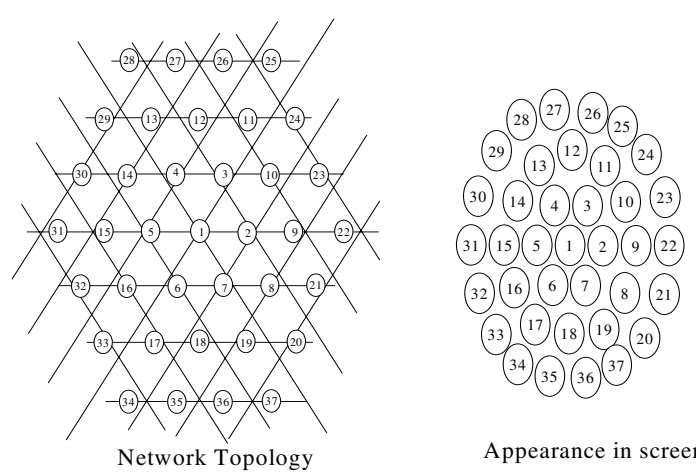

Figure 12: Network Model

\subsection{Simulation Setup and results}

We ran the simulation on a selected hexagonal mesh network. We have chosen this network for simplicity. To show the division and fusion within the community through the simulation time, we have displayed the community as shown in figure 12. Every 5 units of time (t), new member joins the community network. The community size grows to 720 members within about 3600 units of time. Figure 13 shows the growing of the community network during the simulation time. In this figure, members in same sub-community have same color and leaders have deep color. When the growing process stops, the number of sub-communities converges to steady state (about 25 sub-communities). The transmission delay d of each link is about one unit of time. The simulation parameters are tabulated in table 1.

Figure 14 plots the variations of the simulation time with the community communication reliability and the number of members in the community. It shows the effectiveness of the proposed technologies that improve the reliability with the growth of the community. The division, fusion, transition and adaptation technologies are used to adapt the changes of the community size to improve the reliability of the community communication. Figure 14 shows that the reliability degrades at some instances of time and then increases at others instances of time. We argued that to the proposed technologies adapt the changes of the community size. Thus, the reliability is improved.

Table 1: Simulation parameter

\begin{tabular}{|l|c|}
\hline \multicolumn{1}{|c|}{ Items } & $\begin{array}{c}\text { Value } \\
(\mathrm{t}: \text { logical time })\end{array}$ \\
\hline Cycle of operation in nodes & $10[\mathrm{t}]$ \\
\hline Transmission delay $(\mathrm{d})$ & $1[\mathrm{t}]$ \\
\hline Waiting time $\left(\mathrm{WT}_{\text {sub }}\right)$ & $100[\mathrm{t}]$ \\
\hline Cycle to reset counter of received messages $\left(\mathrm{T}_{\text {cycle }}\right)$. & $50[\mathrm{t}]$ \\
\hline
\end{tabular}



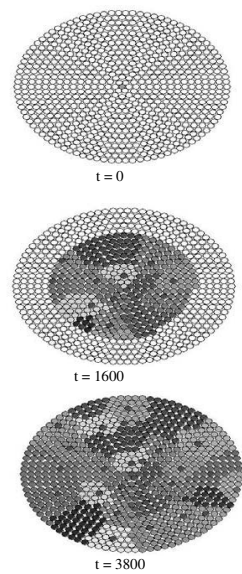
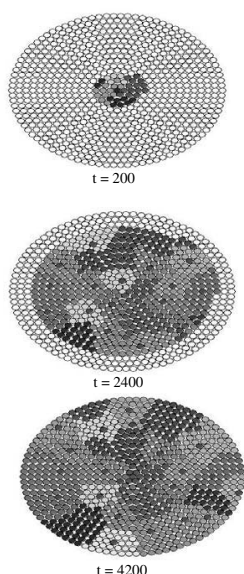

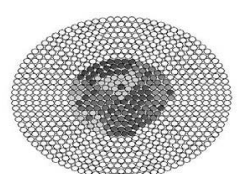

$t=800$
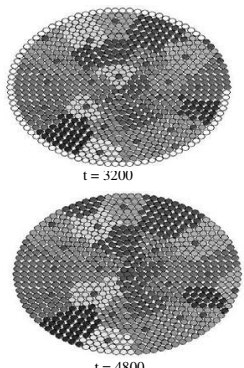

$\mathrm{t}=4800$
Figure 13: Simulation results community growing phases

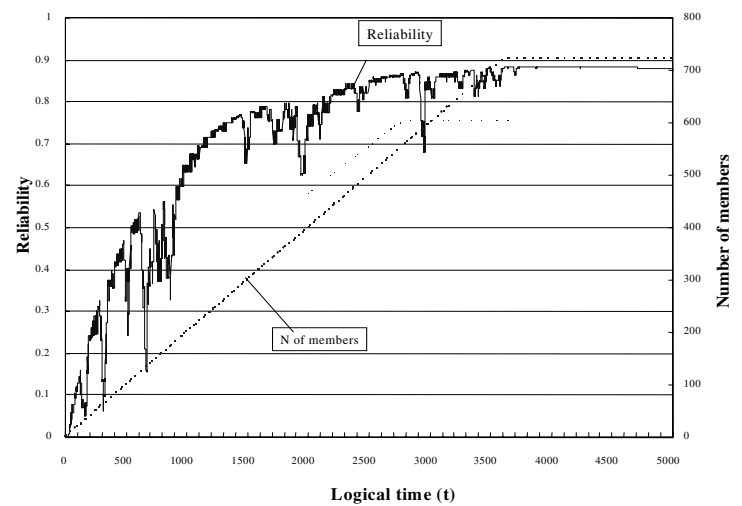

Figure 14: Simulation results of reliability improvements

\section{Conclusion}

It has been increasing even more that the requirement for mobile commerce to provide the reliably services reflecting the users' preference under the evolving market. Users would like to utilize the appropriate services reflecting their location, time and preference. SP would like to grasp the current users' requirements in the area based on each service high reliably to customize its service.

In order to satisfy both the users and SP's requirements the Service-Oriented Community system is proposed. It provides three-dimensional service awareness (location, time and user demand). The role of the service-oriented community system is to reliably send the right information to the right user. The community members (SPs and users) cooperate with one another to get mutual benefits like social community. Consequently, SP can grasp the current requirements by collecting the users' marketing information in the community area reliably. Furthermore, the users and the SP can utilize and provide stable services. In this paper, we have proposed the autonomous decentralized construction technology to achieve reliable communication among the community members. To improve the reliability, we have organized community into sub-communities. When the community growth the reliability may descended. Thus, it is highly required to propose technologies that adapt the changes of the community size to improve the reliability. We have proposed autonomous division, fusion, transition and adaptation technologies to do so.

We have simulated those technologies and the results show the improvements of the reliability. Those technologies assure the reliability with the increasing of the number of members. Thus, an essential component of the modern high-assurance (reliability) in service-oriented community system is achieved.

\section{References}

[1] J. Hightower and G. Borriello, "Location Systems for Ubiquitous Computing," IEEE Computer, vol.34, no.8, pp.57-66, 2001.

[2] N. Marmasse and C. Schmandt, "Location-Aware Information Delivery with ComMotion," HUC2000, LNCS1927, pp.157-171, Springer-Verlag Berlin, 2000.

[3] Y-C. Tseng, W-H. Liao and C-M. Chao, "Location Awareness in Ad-Hoc Wireless Mobile Networks," IEEE Computer, vol.34, no.6, pp.46-52, 2001.

[4] K. Mori, "Towards integrated methods for high assurance systems," IEEE Computer, vol. 31, No. 4, PP. 32-34, 1998

[5] M-A. Dru, S. Saada, "Location-based mobile services: the essentials," Alcatel Telecommunications Review, $1^{\text {st }}$ Quarter 2001, p71-76.

[6] K. Mori, "Autonomous Decentralized System: Concept, Data Field Architecture and Future Trends", Proc. of ISADS93, pp28-34, March 1993.

[7] Takanori Ono, Khaled Ragab, Naohiro Kaji and Kinji Mori, "Service Oriented Communication Technology for Achieving Assurance," In IEEE Proc. Of the $22^{\text {nd }}$ Int. Conf. In Distributed Computing Systems Workshops (ICDCSW'02), 2002.

[8] K. Ragab N. Kaji, K. Mori, "Scalable Multilateral Autonomous Decentralized Community Communication Technique for Large-Scale Information Systems", IEICE Transaction on Comm., Vol. E87-B, No. 3, March 2004.

[9] Naohiro Kaji, Khaled Ragab, Takanori Ono and Kinji Mori, "Autonomous Cooperation Technologies for Achieving Real time Property and Fault Tolerance in Service Oriented Community System," In IEEE Proc. Of the $23^{\text {nd }}$ Int. Conf. In Distributed Computing Systems Workshops (ICDCSW'03), 2003. 